\title{
Effects of Nanoceria on Human Platelet Functions and Blood Coagulation
}

\author{
Jyotsna Kailashiya (D), Debabrata Dash (1D)
}

Centre for Advanced Research on Platelet Signalling \& Thrombosis Biology, Department of Biochemistry, Institute of Medical Sciences, Banaras Hindu University, Varanasi, Uttar Pradesh, India

Correspondence: Debabrata Dash, Centre for Advanced Research on Platelet Signalling \& Thrombosis Biology, Department of Biochemistry, Institute of Medical Sciences, Banaras Hindu University, Varanasi, Uttar Pradesh, India, Email ddash.biochem@gmail.com

Purpose: Cerium oxide nanoparticles (nanoceria) exist in either 3+ or 4+ oxidation state with interesting redox properties and exhibit both oxidant and antioxidant attributes based on concentration, environment and $\mathrm{pH}$. Thanks to their wide spread use in fuel, cosmetics and other industries as well as in biomedical field, nanoceria particles get released into environment with risk of significant human exposure, and thus necessitate careful examination and optimization of their biomedical applications.

Methods: This research was planned to explore effects of nanoceria on human platelet functions and whole blood coagulation. Platelets and whole blood from volunteer healthy donors were treated with different doses of nanoceria and various platelet functions, fibrin polymerization and blood coagulation were tested.

Results: Nanoceria particles were found to reduce platelet aggregation and secretion of granule contents in a dose-dependent manner, impaired fibrin polymerization and retraction of fibrin-rich thrombi, associated with reduced cytosolic filamentous actin. Remarkably, nanoceria instigated early clot formation in whole blood attributable to putative activation of coagulation cascade.

Conclusion: Above observations provide cautionary framework to critically re-evaluate and accordingly plan biomedical applications of nanoceria.

Keywords: cerium oxide nanoparticles, fibrin polymerization, human platelets, platelet aggregation, thromboelastogram

\section{Introduction}

Cerium, belonging to lanthanide series, is a rare earth metal bearing atomic number 58. Nanoparticles of cerium oxide (nanoceria) are a mixture of $\mathrm{CeO}_{2}$ and $\mathrm{Ce}_{2} \mathrm{O}^{1,2}$ carrying $3+$ and $4+$ oxidation states on their surface, which endow them with interesting redox properties. Techniques like green synthesis, hydrothermal, solvothermal, aqueous precipitation, reversed micelles, thermal decomposition and flame spray have been developed to synthesize nanoceria while maintaining control over its size and properties. ${ }^{1-3}$

Nanoceria are being widely employed in areas of chemical mechanical polishing/planarization, cosmetics, fuel oxidation catalysis, corrosion protection, solar cells and automotive exhaust treatment. ${ }^{1,2}$ Interestingly, nanoceria also exhibit bio-relevant properties like mimicking activities of superoxide dismutase, peroxidase, catalase, oxidase and phosphatase, and scavenging hydroxyl radicals, nitric oxide radicals and peroxynitrite. ${ }^{1}$ Redox properties of nanoceria have been proposed to be utilized for therapeutic applications like their superoxide dismutase property for therapy for pancreatic inflammation (pancreatitis), ${ }^{4}$ protection of germ line cells from damaging effects of irradiation in mice, ${ }^{5}$ ROS scavenging and anti-inflammatory effects for rheumatoid arthritis treatment. ${ }^{6}$ Function of nanoceria is attributable to thermodynamically favourable redox cycling between $3+$ and $4+$ states and its unique ability to exchange oxygen. ${ }^{1}$ It can have dual role as an oxidation as well as reduction catalyst, depending on the reaction conditions and surrounding microenvironment. ${ }^{3}$ Nanoceria have been reported to possess pro-oxidant properties at lower $\mathrm{pH}$ and high concentrations, and have shown potential toxicity based on synthesis method, concentration, and exposure time. ${ }^{7}$

As cerium is neither naturally found in the human body nor has it any known excretion mechanism, exposure to cerium can lead to local or systemic toxicity. ${ }^{1}$ In most cases nanoceria have been reported to exhibit beneficial effects like scavenger of reactive oxygen species and regarded as a potential nanomaterial for biomedical applications. However, 


\section{Graphical Abstract}

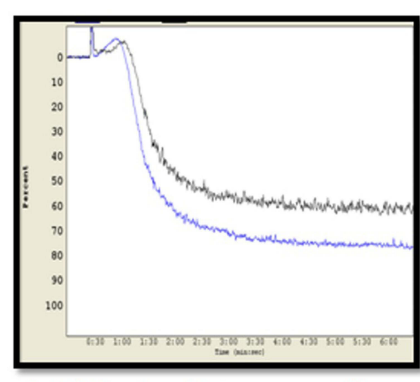

Inhibit platelet aggregation

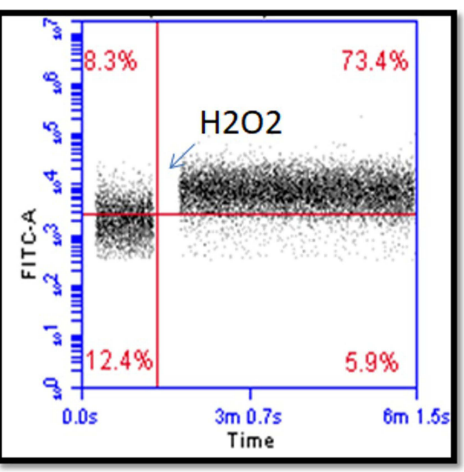

Antioxidant property

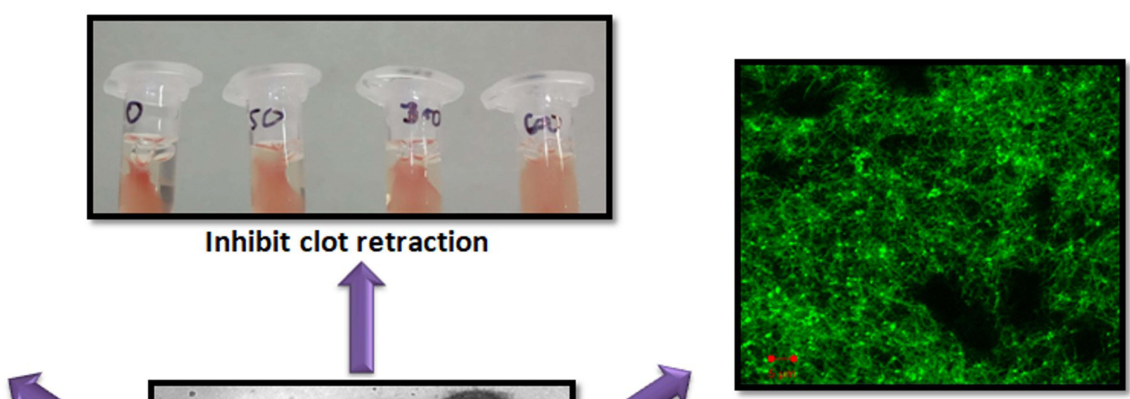

Disrupt fibrin polymerization

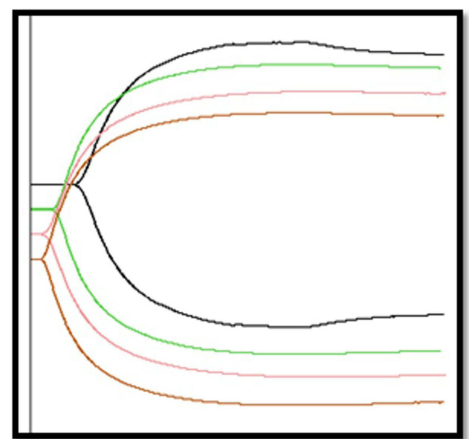

Accelerate whole blood coagulation

several reports to the contrary have alluded to pro-oxidant effect of nanoceria associated with DNA damage, dephosphorylation of substrates, aberrant cell signalling, apoptosis, and alterations in the transcription and post-translational modifications based on in vitro studies. ${ }^{3,8-10}$

Nanoceria have been reported to induce pulmonary inflammation, myocardial fibroblast proliferation, phospholipidosis and release of pro-inflammatory cytokines in laboratory animals. ${ }^{3,11}$ Intravenous (IV)/intra-peritoneal (IP) administration in mice led to deposition of the nanoparticles in organs like spleen, liver, lungs and kidneys, ${ }^{12}$ and incidence of pulmonary and systemic inflammation following intratracheal instillation. ${ }^{13}$ IV and IP administration of nanoceria, also resulted in elevated WBC counts in mice, ${ }^{12}$ and hepatic injury in rats after single vascular infusion. ${ }^{14}$ A previous study on hemocompatibility of nanoceria has reported neither hemolysis nor any significant effect on whole blood aggregation by ADP, though shortening of plasma recalcification time (PRT) was observed at $50 \mu \mathrm{g} / \mathrm{mL}$ dose. $^{15}$

Above data necessitate careful and detailed examination, and optimization of biomedical applications of nanoceria, that are also used as diesel fuel catalyst with potential risk of air emission. ${ }^{1,3}$ Human exposure of nanoceria is mostly through inhalation ${ }^{2}$ and they can easily access blood stream impacting function of circulating cells and plasma enzymes. Platelets are highly sensitive to environment that play critical role in hemostasis and arterial thrombosis, ${ }^{16}$ leading to 
serious pathologies like acute myocardial infarction and ischemic stroke. Thus, this research was planned to study effects of nanoceria on human platelet functions and thrombogenesis.

\section{Materials and Methods}

Cerium oxide nano powder (\#544841), phalloidin-FITC, 2',7'-dichlorodihydrofluorescein diacetate $\left(\mathrm{H}_{2} \mathrm{DCF}-\mathrm{DA}\right)$, purified fibrinogen from human plasma, carbonyl cyanide m-chlorophenyl hydrazone (CCCP), prostaglandin E-1 (PGE-1) and thrombin were purchased from Sigma. Collagen and Chrono-lume luciferin luciferase reagent were products of Chrono-log. Mitotracker red, MitoSOX red, Calcein-AM, Fluo-4-AM and Alexa Fluor 488-conjugated human fibrinogen were from Invitrogen. FITC-conjugated annexin V, PAC1 and anti-CD62P antibodies were from BD BioSciences. Hydrogen peroxide was from Thermo Fisher and trypan blue was from SRL laboratories. All other reagents used were of analytical grade. Type I deionized water $(18.2 \mathrm{M} \Omega \cdot \mathrm{cm}$, Millipore) has been used throughout the experiments.

\section{Preparation of Nanoceria Suspension}

Nanoceria powder was procured commercially from M/S Sigma (\#544841) for the present study as it exhibits suitable redox properties. ${ }^{15}$ The nano powder was mixed with type I Milli Q-grade water at $5 \mathrm{mg} / \mathrm{mL}$ concentration. Mixture was sonicated with a probe sonicator (Misonix, USA) for 3 cycles of 5 min each (with $30 \mathrm{sec}$ intervals in between), which resulted in milky white suspension. Larger particles and aggregates in the suspension were settled down by centrifugation at $200 \mathrm{~g}$ for $5 \mathrm{~min}$. Supernatant was collected in fresh vial and stored at $2-8{ }^{\circ} \mathrm{C}$ until further use.

\section{Characterization of Nanoceria}

Particles in nanoceria suspension were analyzed with Nanoparticle Tracking Analyzer (NTA) following 1:1000 dilution. Both capture and analysis were performed employing NanoSight LM10 (Malvern) and NTA 2.3 analytical software, which provide an estimate of nanoparticle size and concentration in sample.

\section{Preparation of Platelet-Rich Plasma (PRP) and Washed Platelets (WP)}

Blood was collected from healthy volunteers, strictly as per recommendations of the Institutional Ethical Committee of Institute of Medical Sciences, Banaras Hindu University. Purpose of blood collection and research was explained to all volunteers and their consent was recorded on signed consent forms. Collected blood was centrifuged at $200 \mathrm{~g}$ for $10 \mathrm{~min}$. Platelet-rich plasma (PRP) thus obtained (as supernatant) was incubated with $1 \mathrm{mM}$ acetylsalicylic acid for $15 \mathrm{~min}$ at $37^{\circ} \mathrm{C}$. After addition of EDTA $(5 \mathrm{mM})$, platelets were sedimented by centrifugation at $600 \mathrm{~g}$ for $10 \mathrm{~min}$. Cells were washed in buffer A (20 mM HEPES, $138 \mathrm{mM} \mathrm{NaCl}, 2.9 \mathrm{mM} \mathrm{KCl}, 1 \mathrm{mM} \mathrm{MgCl} 2,0.36 \mathrm{mM} \mathrm{NaH} \mathrm{PO}_{4}, 1 \mathrm{mM}$ EGTA, supplemented with $5.5 \mathrm{mM}$ glucose and were finally resuspended in buffer B (pH 7.4), which was the same as buffer A but without EGTA. The final cell count was adjusted to $2-4 \times 10^{8} / \mathrm{mL}$ with cell counter (Multisizer 4, Beckman Coulter). The study methodologies conformed to the standards set by the Declaration of Helsinki. All steps were carried out under sterile conditions. Precautions were taken to maintain the cells in resting condition to prevent spontaneous platelet activation that include storage in polypropylene plasticware at $22-24{ }^{\circ} \mathrm{C}$, gentle handling to avoid swirl in the cell suspension and use of platelets within $4 \mathrm{~h}$ of harvesting.

\section{Nanoceria Treatment}

Nanoceria were added to platelet suspension at ratios of 50,300 or 600 cerium oxide nanoparticles per platelet, designated as NC50, NC300 or NC600, respectively (Supplementary Table 1), and incubated at $37{ }^{\circ} \mathrm{C}$ for 30 min to allow uptake of nanoparticles by platelets. Ultrapure type I deionized Milli-Q grade water $(18.2 \mathrm{M} \Omega \cdot \mathrm{cm})$ was used as vehicle in control samples.

\section{Flow Cytometry}

Platelet suspensions, either with or without nanoceria treatment, were diluted with sheath fluid (1:5) and analyzed with a flow cytometer (Becton Dickinson, model Accuri C6). Platelet gate was drawn in log scale forward (FSC) and side (SSC) scatter dot plots and 10,000 events were acquired for each sample. Following blocking with 2\% BSA samples 
were treated with either FITC-labelled PAC1, CD62P or annexin V ( $2 \mu \mathrm{L}$ each from stock). For annexin V binding $2 \mathrm{mM}$ $\mathrm{CaCl}_{2}$ was added to WP samples. Samples were also stained with Calcein-AM $(3 \mu \mathrm{g} / \mathrm{mL})$ for cell viability, Fluo-4-AM (1 $\mu \mathrm{M})$ for intracellular calcium, Mitotracker red $(0.5 \mu \mathrm{M})$ for mitochondrial membrane potential, MitoSOX red $(5 \mu \mathrm{M})$ for mitochondrial ROS, FITC-conjugated fibrinogen $(5 \mu \mathrm{g} / \mathrm{mL})$ for fibrinogen binding capacity and trypan blue $(0.002 \%)$ for cell viability and membrane integrity. For study of actin polymerization, platelets were activated with $0.5 \mathrm{U} / \mathrm{mL}$ thrombin for 10 min followed by fixing with $2 \%$ paraformaldehyde. Cells were washed with PBS, permeabilized with $0.1 \%$ Triton $\mathrm{X}-100$, and blocked with BSA (2\%) for $30 \mathrm{~min}$. Intracellular F-actin was stained with phalloidin-FITC (2.5 $\mu \mathrm{M})$ for 30 min, washed with PBS and analyzed by flow cytometry. Intracellular ROS was measured after incubating platelets with $10 \mu \mathrm{M} \mathrm{H} \mathrm{H}_{2}$ DCF-DA in buffer B for 30 min.

\section{Transmission Electron Microscopy}

Platelets were incubated with nanoceria for $30 \mathrm{~min}$ and fixed with Karnovsky`s solution composed of glutaraldehyde (2.5\%) and paraformaldehyde (2\%) in $100 \mathrm{mM}$ phosphate-buffer at $\mathrm{pH} 7.4$. Ultra-thin sections (70 $\mathrm{nm}$ thick) were prepared with an ultramicrotome (UC 7, Leica). Samples were mounted on formvar-coated grids, stained with uranyl acetate and lead citrate, and examined under a transmission electron microscope (Talos, Thermo Fischer Scientific) equipped with field emission gun and CMOS (complementary metal oxide semiconductor) detector operated at $200 \mathrm{KV}$. Images were acquired using TIA (Technai Imaging \& Analysis) software provided with equipment.

\section{Platelet Aggregation and Secretion}

WP or PRP $\left(250 \mu \mathrm{L}, 2 \times 10^{8}\right.$ platelets $\left./ \mathrm{mL}\right)$ were incubated with either nanoceria or vehicle (type I deionized Milli-Q water) for $30 \mathrm{~min}$ at $37^{\circ} \mathrm{C}$. Aggregation was induced with $5 \mu \mathrm{g} / \mathrm{mL}$ collagen (in PRP) or $0.5 \mathrm{U} / \mathrm{mL}$ thrombin (in WP) in a whole blood/optical lumi-aggregometer (Chrono-log model 700-2) as percent change in light transmission, where $100 \%$ refers to transmittance through blank (buffer). Release of adenine nucleotides from platelet dense granules was measured by addition of $15 \mu \mathrm{L}$ Chrono-lume reagent (from stock $0.2 \mu \mathrm{M}$ luciferase/luciferin) to WP immediately before aggregation. Luminescence generated was monitored using the lumi-aggregometer contemporaneous with platelet aggregation measurement.

\section{Clot Retraction Assay}

PRP $(100 \mu \mathrm{L})$, incubated with or without nanoceria at $37{ }^{\circ} \mathrm{C}$, was diluted with $300 \mu \mathrm{L}$ buffer B, to which RBCs $(1 \mu \mathrm{L}$ from sedimented cells after isolation of PRP) and calcium chloride ( $2 \mathrm{mM})$ were added. Clot formation was induced by addition of thrombin $(1 \mathrm{U} / \mathrm{mL})$. After incubation for $1 \mathrm{~h}$ at $37^{\circ} \mathrm{C}$, extent of retraction was evaluated from the volume of fluid squeezed out from the retracted clot, which was carefully aspirated and measured. ${ }^{17}$

\section{Fibrin Polymerization Assay}

Fibrin polymerization was measured by a turbidimetry-based assay. Purified human fibrinogen $(100 \mu \mathrm{L}$ from $2 \mathrm{mg} / \mathrm{mL}$ stock), treated with or without nanoceria, was placed in flat bottom 96-well plate. After incubation of 30 min at $37^{\circ} \mathrm{C}$, polymerization was initiated by addition of $1 \mathrm{U} / \mathrm{mL}$ thrombin and $5 \mathrm{mM} \mathrm{CaCl}_{2}$. Optical density (OD) at $350 \mathrm{~nm}$ was recorded every 10 min interval till $1 \mathrm{~h}$ as a measure of clot formation with a fluorescence multi-mode microplate reader (BioTek model Synergy H1). ${ }^{18}$

For microscopic examination of fibrin polymer, Alexa fluor 488-labelled fibrinogen (5\%) was added to stock fibrinogen. Following treatment with nanoceria or vehicle, $20 \mu \mathrm{L}$ fibrinogen $(0.5 \mathrm{mg} / \mathrm{mL})$ was placed on glass slides. Polymerization was initiated by addition of $1 \mathrm{U} / \mathrm{mL}$ thrombin and $5 \mathrm{mM} \mathrm{CaCl}_{2}$. After $1 \mathrm{~h}$ incubation fibrin clot was washed twice with PBS and cover slip was placed over it. Morphology of fibrin polymers was observed under confocal laser scanning microscope (Zeiss model LSM 700), using 63X oil immersion objective. Images were acquired and analysed employing Zen imaging software. 


\section{Thromboelastography (TEG)}

Coagulation parameters in whole blood were studied by TEG analysis employing thromboelastograph 5000 hemostasis analyzer system (Haemonetics) and TEG analytical software. Whole blood $(1 \mathrm{~mL})$ was incubated either with nanoceria or vehicle, followed by transfer into citrated kaolin tubes with proper mixing. $\mathrm{CaCl}_{2}(20 \mu \mathrm{L}$ from $0.2 \mathrm{M}$ stock) was added to $340 \mu \mathrm{L}$ sample to initiate coagulation cascade. Mixture was placed in disposable TEG cups and data were collected as per manufacturer instructions until maximum amplitude was reached or $60 \mathrm{~min}$ had elapsed.

This study was approved by ethical committee of Institute of Medical Sciences, Banaras Hindu University. Experiments were repeated for 3 or more times and data was compared using analysis of variance (ANOVA) with post hoc tests. $\mathrm{P}$ value less than 0.05 was considered significant. Symbol * indicates $\mathrm{p}$ value $<0.05$, ** indicates $\mathrm{p}$ value $<0.01$ and $* * *$ indicates $\mathrm{p}$ value $<0.001$.

\section{Results}

\section{Nanoceria Particle Characterization}

NTA analysis was consistent with presence of nanoceria particles ranging from 30-790 nm (hydrodynamic size) in the suspension, majority of which were within size range of 98-133 nm (Supplementary Figure 1). Average particle count in $5 \mathrm{mg} / \mathrm{mL}$ nanoceria suspension was found to be $18.33 \pm 0.38 \times 10^{11} / \mathrm{mL}(\mathrm{n}=4)$, which was adjusted as per experimental requirement (Supplementary Table 1). Morphology and surface characterization of particles in preparation have been described earlier. ${ }^{15}$

\section{Nanoceria Uptake by Platelets}

Flow cytometry of washed human platelets incubated with increasing concentrations (NC300 and NC600) of nanoceria demonstrated dose-dependent rise in side scatter (Figure 1A-C), consistent with enhanced cellular complexity in following nanoparticle uptake. Platelets in PRP also exhibited significant escalation in SSC when incubated with nanoceria at NC600 dose (Supplementary Figure 2A). No significant change was observed in forward scatter (data not shown). TEM confirmed considerable uptake of nanoceria by platelets and their localization in cytoplasm as dark particles (indicated by arrows in Figure 1D).

\section{Nanoceria Inhibited Agonist-Induced Platelet Functions}

Light transmittance based assay method was used for platelet aggregation study, where aggregation of platelets results in clearance of fluid and increased transmittance of light, which is reflected in percentage of light transmission, representing platelet aggregation in real time. Nanoceria significantly inhibited thrombin $(0.5 \mathrm{U} / \mathrm{mL})$-induced aggregation of washed human platelets in a concentration-dependent manner (Figure 2A and B). Nanoceria at NC600 concentration, too, abrogated collagen $(5 \mu \mathrm{g} / \mathrm{mL})$-induced aggregation of platelets in PRP (Supplementary Figure 2B and $\underline{\text { ) }}$. Contemporaneous with aggregation, nanoceria inhibited release of ATP from platelet dense granules (Figure 2A and C) and P-selectin from alpha granules (Figure 2D) in thrombin-stimulated platelets. Retraction of fibrin-rich clot is an important function of platelets that restricts access of plasminogen activator into fibrin mesh, thus preventing thrombolysis and contributing to clot stability. Clot retraction assay is based on quantifying volume of free fluid that has been cleared after platelet and fibrin rich clot has formed and retracted in samples. Remarkably, prior exposure to nanoceria significantly impaired clot retraction triggered by $1 \mathrm{U} / \mathrm{mL}$ thrombin (Figure 2E and F). Nanoceria also brought about significant reduction in level of ROS both in resting as well as activated platelets, and quenched the rise in ROS elicited in presence of $\mathrm{H}_{2} \mathrm{O}_{2}$ (Figure 3).

\section{Nanoceria Inhibited Thrombin-Induced Actin Polymerization in Washed Platelets}

As agonist-induced platelet functions like aggregation, secretion and clot retraction demand extensive reorganization of actin-based cytoskeleton, we next investigated effect of nanoceria on stability of filamentous actin (F-actin) in thrombinstimulated platelets from extent of staining with phalloidin-FITC. F-actin content was significantly dropped by $23 \%$ when platelets were exposed to nanoceria at dose NC600 (Figure 4A and B), which was reflective of lack of actin polymerization elicited in presence of nanoparticles resulting in attenuated platelet functions. 
A

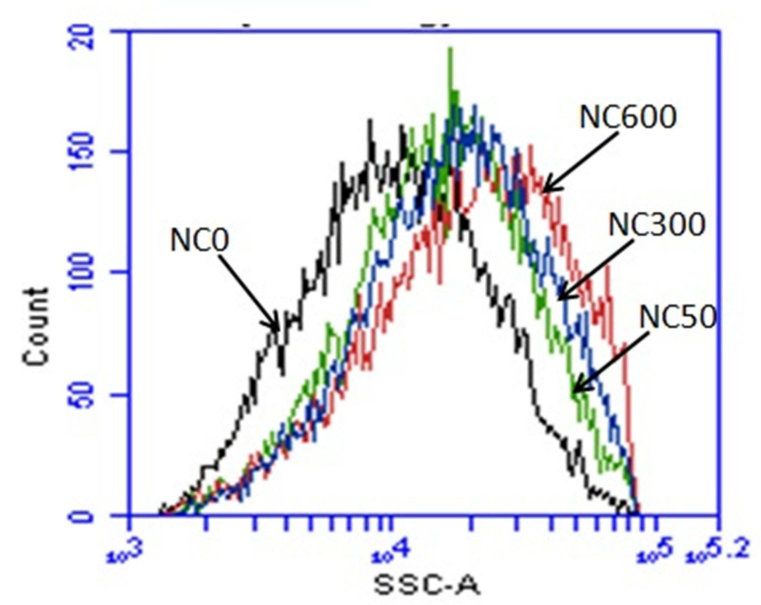

B

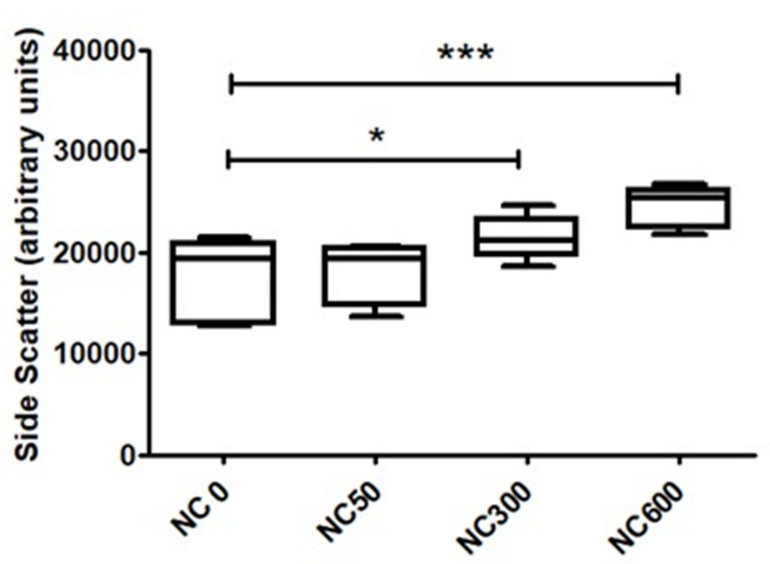

C

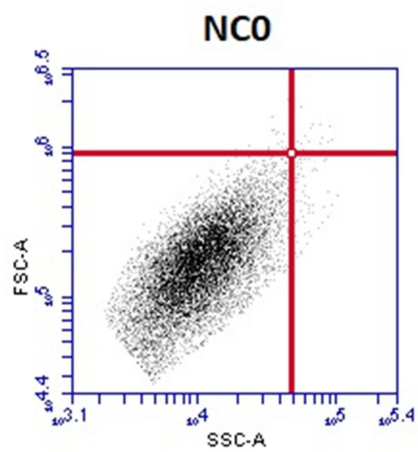

NC50

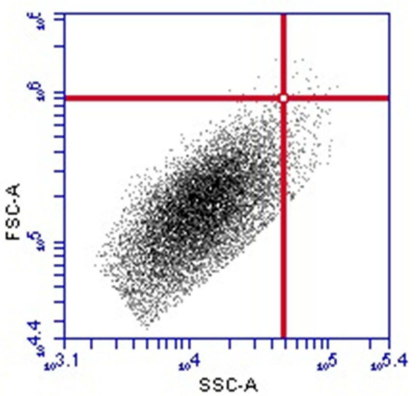

NC300

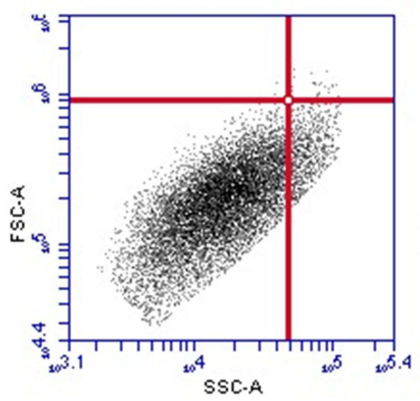

NC600

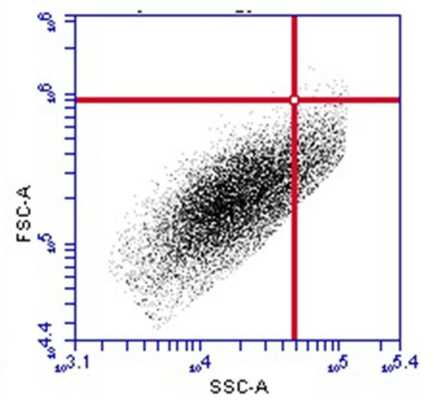

D

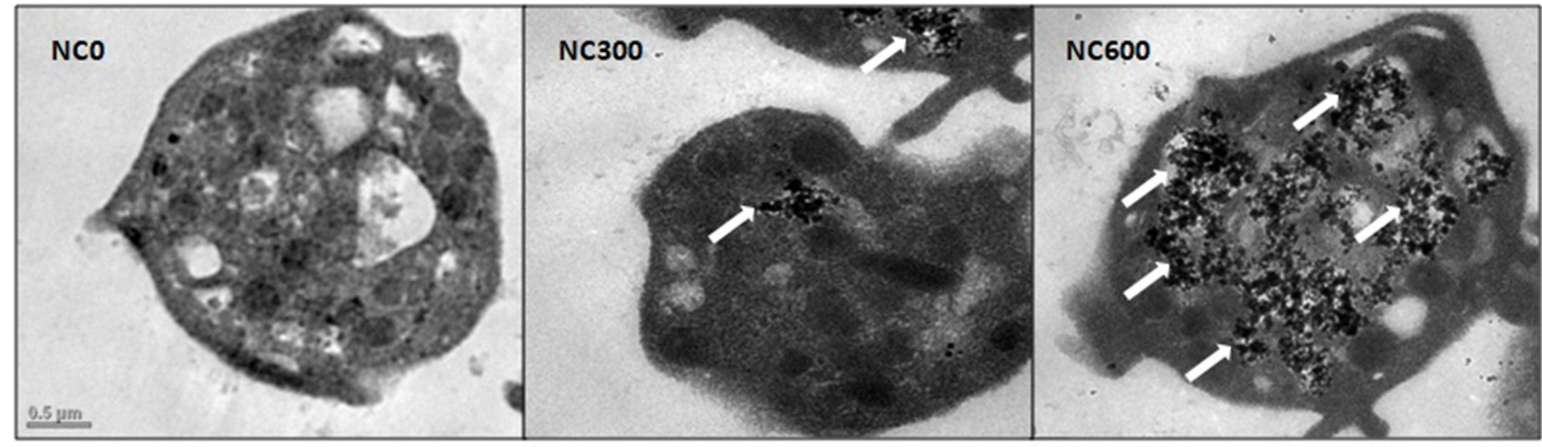

Figure I Flow cytometry of washed platelets, presented as histograms (A), statistical presentation as box plots (B) and scatter plots (C), following 30 min incubation with increasing concentrations of nanoceria, showing dose-dependent rise in side scatter (SSC) ( $n=5$, repeated measure ANOVA). (D) TEM images showing uptake and intracellular localization of nanoceria particles (marked with arrows) in platelet cytosol. ( $\left.{ }^{*} p<0.05, *^{* * *} p<0.00 \mathrm{I}\right)$.

\section{Nanoceria Modulate Kinetics of Clot Formation and Clot Architecture}

Soluble fibrinogen is cleaved by thrombin in presence of calcium to generate fibrin monomers, which soon polymerize to form insoluble fibrin mesh/clot. Process of fibrin mesh formation can be assayed by turbidimetry as fibrin polymers occlude the light path in a spectrophotometer. For quantitative analysis to detect effects of nanoceria, fibrin polymerization was induced by addition of thrombin to solution of human fibrinogen in presence or absence of nanoceria and rise in $\mathrm{OD}$ at $350 \mathrm{~nm}$ was recorded as a measure of ensuing clot formation. Nanoceria significantly impeded fibrin polymerization at all the doses tested (Figure 5A and B). This led us to examine clot architecture by allowing Alexa fluor 488labelled fibrinogen to polymerize in presence nanoparticles, followed by confocal microscopy. Strikingly, nanoceria 

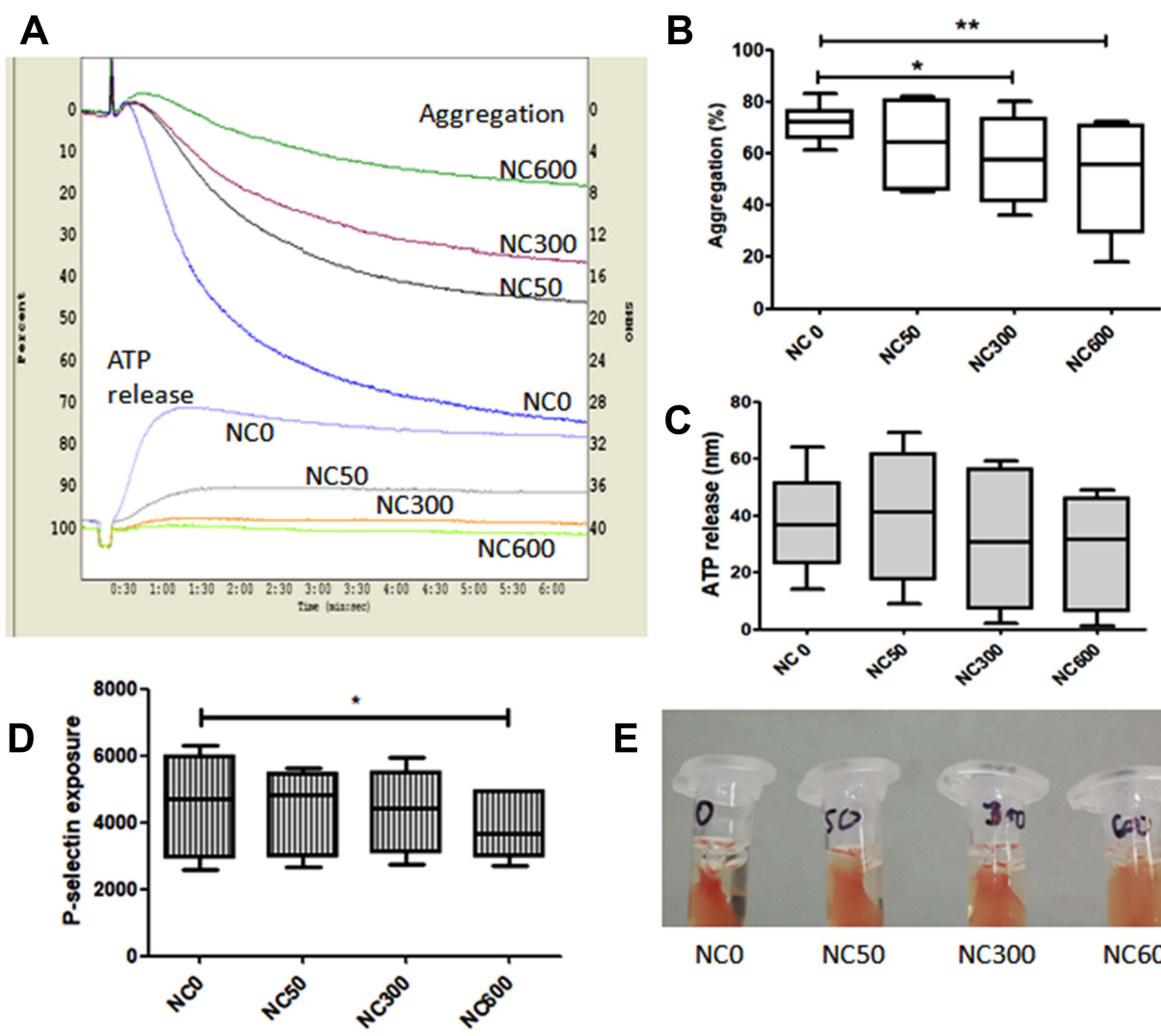

E
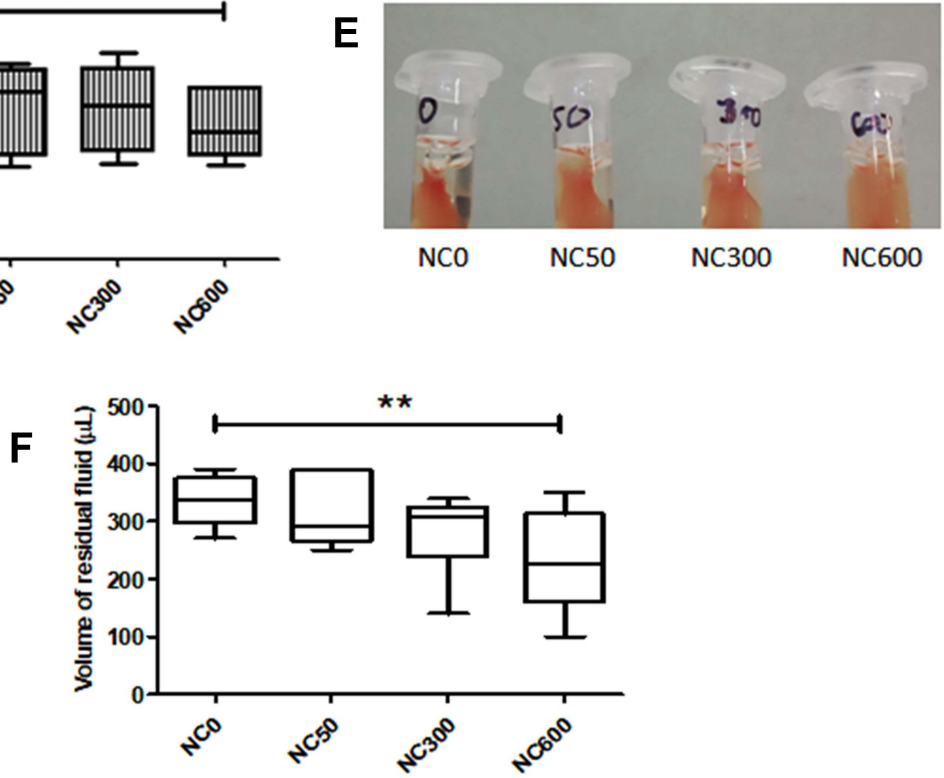

Figure 2 Thrombin $(0.5 \mathrm{U} / \mathrm{mL})$-induced aggregation (A and B), ATP secretion $(\mathbf{C})$ and P-selectin exposure (D) in washed platelets $\left(2 \times 10^{8}\right.$ per $\left.\mathrm{mL}\right)$ incubated for $30 \mathrm{~min}$ with and without nanoceria $(n=6$, repeated measure ANOVA). ( $E$ and $\mathbf{F})$ thrombin-induced clot retraction in PRP $(n=5$, Repeated measure $A N O V A)$. $(* p<0.05$, **p $<0.01)$.

brought about substantial disruption in morphology of fibrin polymer (Figure 5C) as the clot transformed to become more granular and non-uniform in presence of nanoceria. Keeping with these observations, thromboelastography of whole blood in presence of nanoceria revealed significantly reduced reaction time $(\mathrm{R})$ and increased kinetics of clot formation ( $\alpha$-angle) that was reflective of rapid induction of coagulation cascade leading to altered clot architecture (Figure 5D-F). However, nanoceria did not have any effect on cell viability, intracellular calcium level or mitochondrial membrane potential in washed platelets and PRP ( $\underline{\text { Supplementary Figures } 3}$ and $\underline{4})$.

\section{Discussion}

In order to exploit their attributes like redox and enzyme mimetic properties, nanoceria are being widely explored and employed in industries as well as biomedical fields like ROS scavenging, anti-inflammatory and anticancer therapies, ${ }^{1,2}$ thus leading to increasing risk of human exposure. Nanoceria have been reported to be toxic to human lymphocytes and monocytes $^{8}$ and accumulates in organs like liver and spleen. ${ }^{3}$ Platelets are highly sensitive cells circulating in blood that play critical role in hemostasis and development of serious occlusive pathologies like arterial thrombosis. As 
A

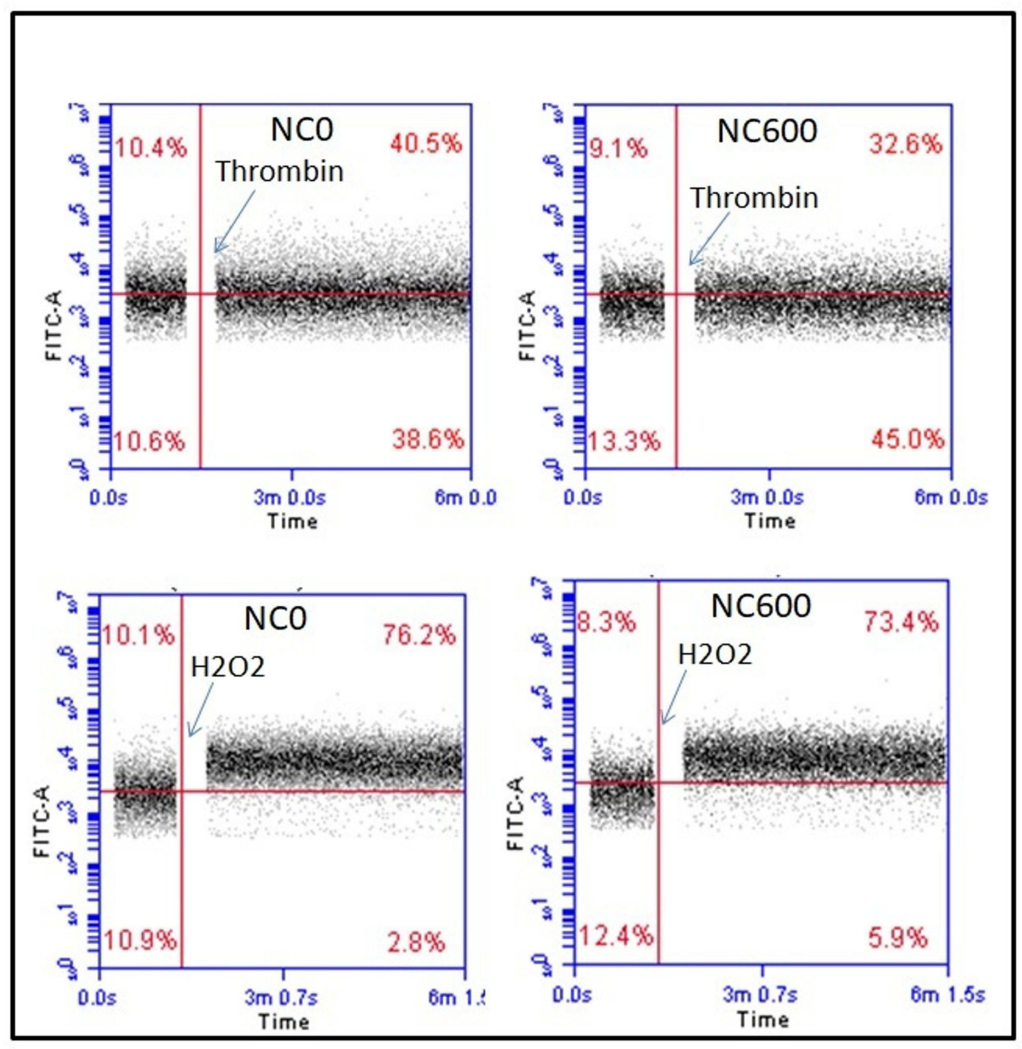

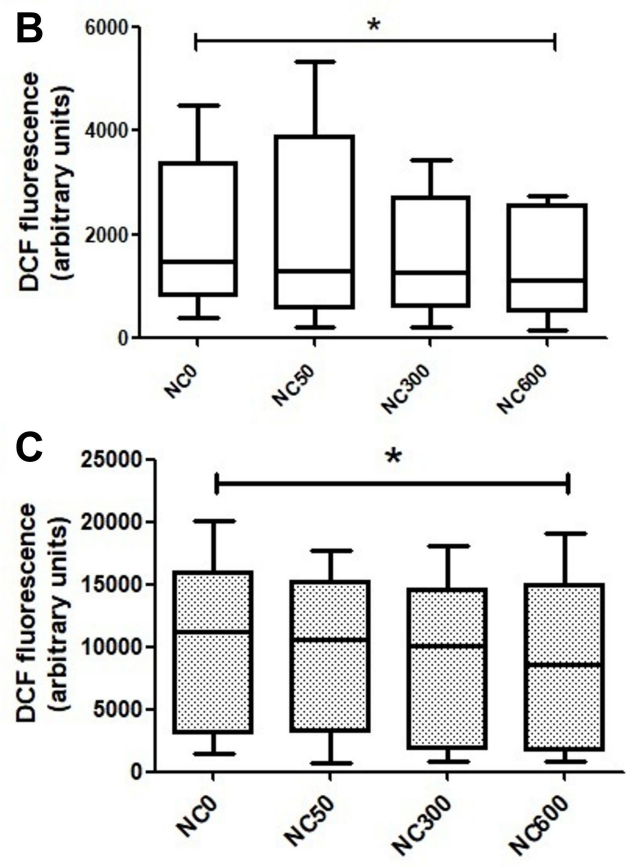

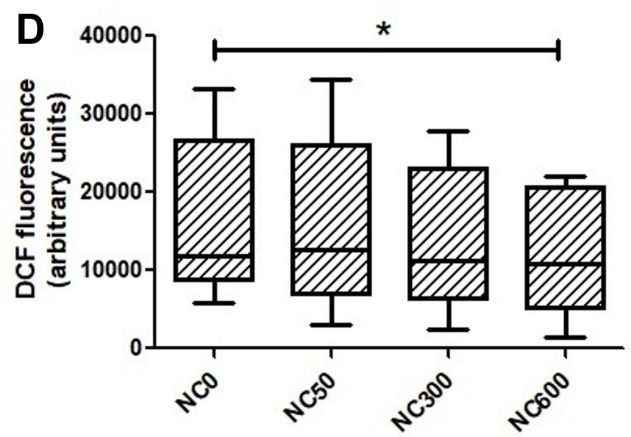

Figure 3 (A) Intracellular ROS in $\mathrm{H}_{2}$ DCF-DA-stained platelets in absence (NC0) or presence of nanoceria (NC600) analysed by flow cytometry. PRP samples were incubated for 30 min with varying concentrations of nanoceria, followed by washing and staining with $\mathrm{H}_{2}$ DCF-DA and treatment with thrombin or $\mathrm{H}_{2} \mathrm{O}_{2}$, as indicated. (B-D) box plots representing intracellular ROS level in resting platelets $(B)(n=10)$, as well as in platelets following 5 min exposure with either $0.5 \mathrm{U} / \mathrm{mL}$ thrombin $(\mathbf{C})(n=5)$ or 50 $\mu \mathrm{M} \mathrm{H}_{2} \mathrm{O}_{2}(\mathrm{n}=5)$, respectively (repeated measure ANOVA). ( $\left.{ }^{*} \mathrm{p}<0.05\right)$.

A

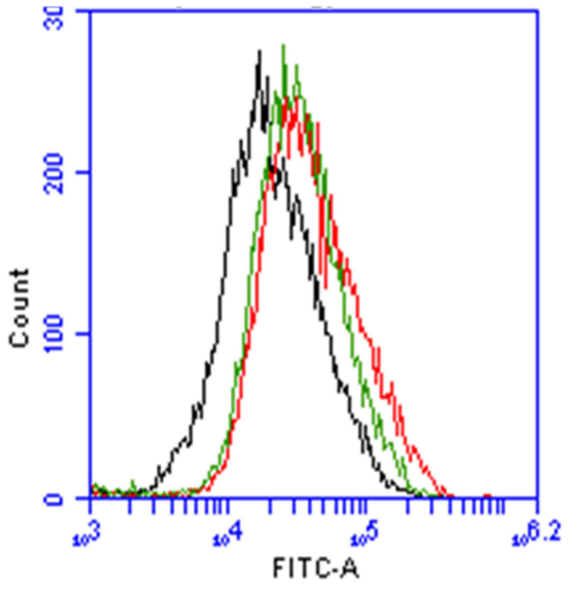

B

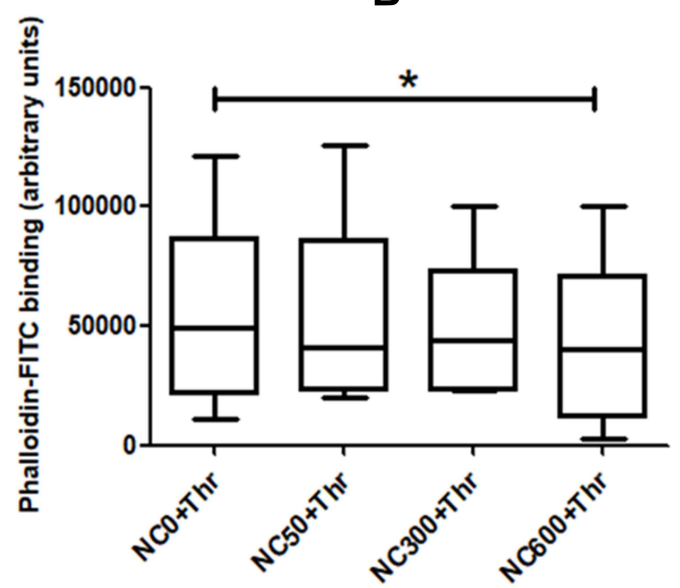

Figure 4 (A) decrease in F-actin content by nanoceria (dose NC600) in thrombin $(0.5 \mathrm{U} / \mathrm{mL})$-stimulated washed platelets $\left(2 \times 10^{8}\right.$ per $\left.\mathrm{mL}\right)$ treated with phalloidin-FITC $(2.5$ $\mu \mathrm{M})$ and analyzed by flow cytometry. Black tracing, resting platelets; red tracing, thrombin-stimulated platelets without nanoceria; and green tracing, thrombin-stimulated platelets after nanoceria (NC600) treatment. (B) box plots representing F-actin content as indicated ( $n=5$, repeated measure ANOVA). $\left({ }^{*} p<0.05\right)$. 
A

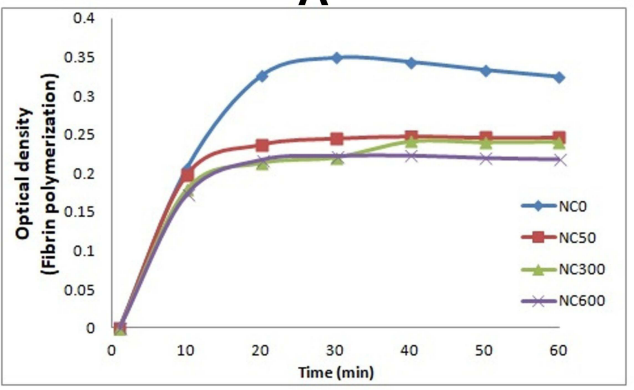

B

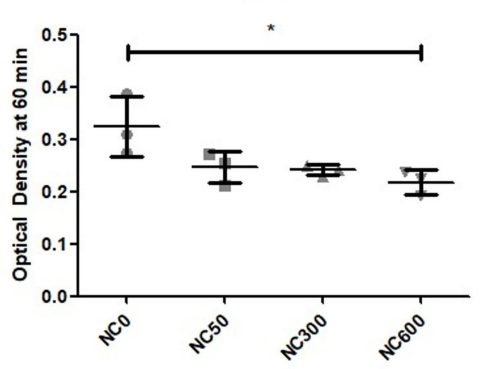

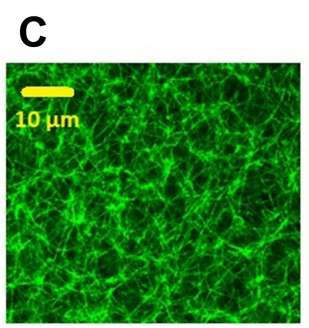

Low magnification

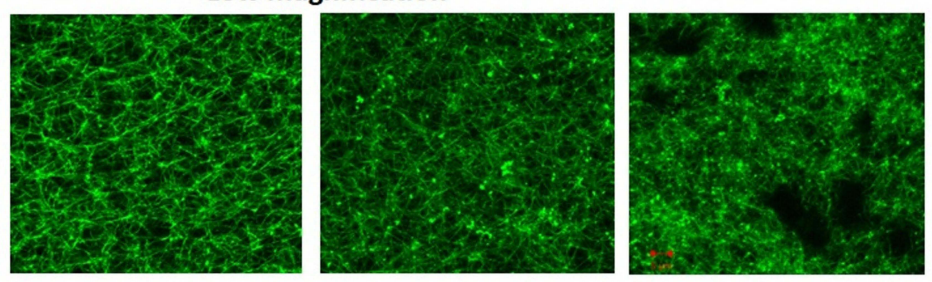

High magnification

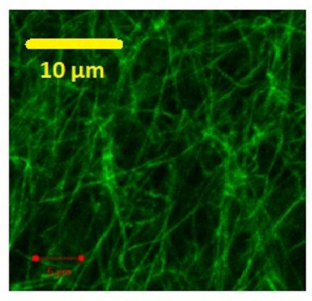

NCO

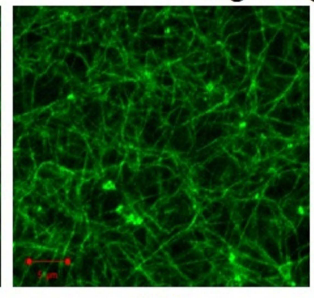

NC50

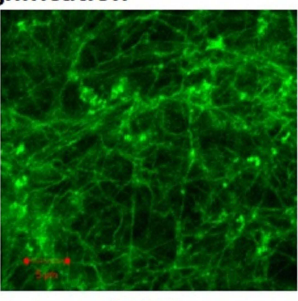

NC300

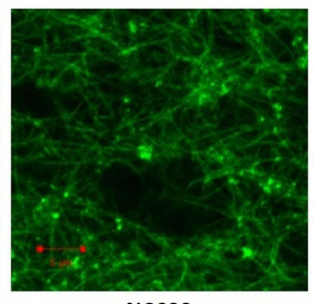

NC600

D

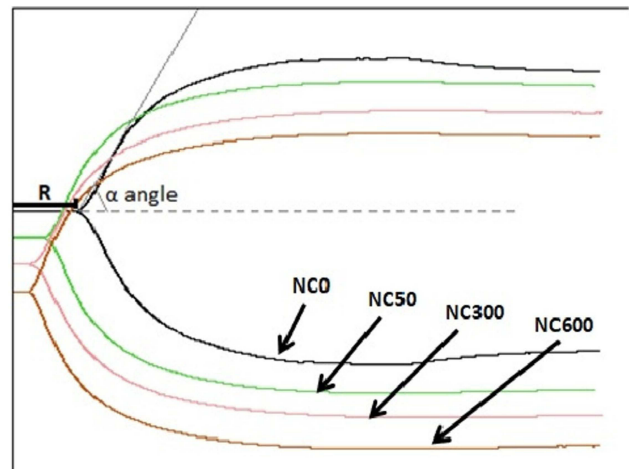

10 millimeters

E
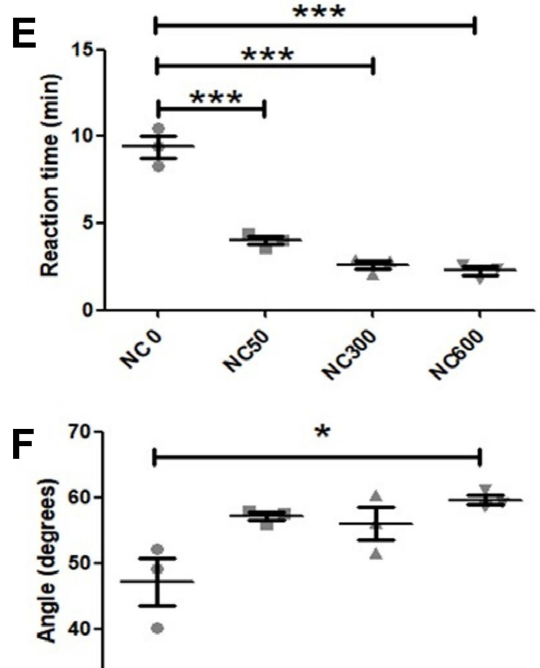

Figure 5 (A and B) nanoceria inhibited fibrin polymerization. (C) nanoceria disrupted normal morphology of fibrin polymer. (D-F) thromboelastogram of kaolin-stimulated citrated whole blood, without and with increasing doses of nanoceria, exhibiting reduction in reaction time and rise in speed of clot formation (Figures are representative of 3 independent experiments, compared by repeated measure ANOVA). $\left({ }^{*} p<0.05, * * * p<0.00 I\right)$.

nanoparticles accessing blood stream would encounter circulating platelets early, we undertook this study to explore their impact on platelet reactivity and coagulation system.

Nanoceria were found to be readily taken up by human platelets, which was reflected in dose-dependent rise in side scatter events in flow cytometry and electron microscopy (Figure 1 and Supplementary Figure 2A). Our observation of rise in platelet SSC following nanoceria treatment is supported by previous reports of rise in side scatter of various cells after nanoparticle uptake. ${ }^{19-21}$ Platelet aggregation induced by physiological agonists was significantly inhibited in presence of nanoceria. 
Strikingly, higher concentration of nanoceria (NC600) was required to inhibit platelet aggregation in PRP (Supplementary Figure 2B) while a lower dose (NC300) was sufficient to impair aggregatory response in washed platelets (Figure 2B), which could be attributable to interference by plasma proteins in PRP. Interference by biologically derived proteins has already been reported to interfere in cellular nanoparticle interactions. ${ }^{22}$ Nanoceria also reduced agonist-evoked secretion from platelet $\alpha$ and dense granules (Figure 2C and D), and retraction of fibrin-rich clot in concentration-dependent manner (Figure 2E and F). This was associated with significant drop in level of ROS in platelets exposed to nanoceria (Figure 3), though higher dose of nanoceria was required for producing antioxidant effects. ROS lowering effect of nanoceria was observed in PRP samples, and not in platelets suspended in buffer (supplementary Figure 3), which could be attributable to influence of plasma proteins and other molecules in a physiological suspension like plasma. ${ }^{22}$ This observation can be implicated in designing antioxidant therapies in conditions associated with increased ROS stress in platelets, like thrombotic risk and platelet storage lesions. ${ }^{23,24}$ Nanoceria did not affect platelet viability nor mitochondrial function at the concentrations studied (Supplementary Figure 2). An earlier study by Del Turco et al, ${ }^{15}$ however, did not observe notable effect of nanoceria particles on whole blood aggregation induced by ADP. This could be attributable to relatively lower doses of nanoceria used by Del Turco et al (10$50 \mu \mathrm{g} / \mathrm{mL}$ ) than used in the present study $(27-324 \mu \mathrm{g} / \mathrm{mL})$, use of a weak agonist like ADP to induce platelet aggregation and difference in testing methods. Strikingly, nanoscale silver, too, have been demonstrated to possess strong inhibitory effect on platelet activation and clot formation by our group ${ }^{25,26}$ and others ${ }^{27}$ while graphene, the 2-dimensional carbon-based nanomaterial, is a well-established modulator of platelet reactivity and thrombogenicity. ${ }^{28,29}$

Rise in content of F-actin and reorganization of actin-based cytoskeleton underly the pro-thrombotic responses of platelets transformed to "activated" phenotype. ${ }^{30,31}$ Nanoceria prevented actin polymerization in thrombin-stimulated platelets (Figure 4), which would explain attenuation of agonist-induced platelet responses in presence of nanoceria. Nanoceria also inhibited retraction of fibrin-rich clot (Figure 2E and F), which involves interaction between platelet surface integrins $\alpha \mathrm{IIb} \beta 3$ and extracellular fibrin that provokes outside-in signaling with ensuing actomyosin contractility. ${ }^{17}$ Significantly, nanoceria elicited impairment of cell-autonomous fibrin polymerization at various doses tested (Figure 5A and B), which reflected steric hindrance in fibrin oligomer extension into protofibrils imposed by inter-strand localization of nanoparticles. Consistent with this observation, nanoceria brought about substantial disruption in morphology of the clot (Figure 5C) as it transformed to a more granular and non-uniform phenotype in presence of nanoceria. Notably, we have earlier demonstrated negative effects of silver nanoparticles on fibrin polymerization similar to nanoceria. ${ }^{25}$

Blood coagulation is a complex process involving activation of clotting factors on platelet surface leading to fibrin polymerization. ${ }^{32}$ In order to investigate the effect of nanoceria, we induced whole blood coagulation by stimulating kaolin-mediated intrinsic pathway and subjected to thromboelastography. Our results were consistent with reduced reaction time in presence of nanoceria, which initiated clot formation significantly earlier than control samples (Figure 5D-F) underscoring early and rapid activation of clotting factors. These findings supported an earlier study showing reduced PRT by nanoceria. ${ }^{15}$ Thus, our observations are reflective of rapid induction of coagulation cascade in whole blood in presence of nanoceria whereas fibrin polymerization, as studied with purified fibrinogen preparation, was incumbered by the nanoparticle leading to altered clot architecture.

\section{Conclusions}

Higher concentration of nanoceria was required to elicit antioxidant effect in platelets, though coagulation cascade was facilitated in whole blood at relatively lower doses. Thus, it is prudent to consider effective concentration of nanoceria that can elicit the desired effects on platelets and coagulability. As nanoceria exhibited significant impact on various platelet functions, clot morphology and blood coagulation, our observations provide cautionary framework to critically re-evaluate and accordingly design biomedical applications of nanoceria.

\section{Acknowledgments}

This research was supported by grants received by D. Dash from the Indian Council of Medical Research (ICMR) under Nanomedicine Task Force, 'ICMR Centre for Advanced Research' (CAR) scheme, Science and Engineering Research Board (SERB), Government of India, J. C. Bose National Fellowship (SERB) and Humboldt Foundation, Germany. 


\section{Author Contributions}

All authors contributed to data analysis, drafting or revising the article, gave final approval of the version to be published, agreed to the submitted journal, and agree to be accountable for all aspects of the work.

\section{Disclosure}

The authors report no conflicts of interest in this work.

\section{References}

1. Dhall A, Self W. Cerium oxide nanoparticles: a brief review of their synthesis methods and biomedical applications. Antioxidants. $2018 ; 7(8): 97$. doi:10.3390/antiox7080097

2. Rajeshkumar S, Naik P. Synthesis and biomedical applications of cerium oxide nanoparticles - a review. Biotechnol Rep. 2018;17:1-5. doi:10.1016/ j.btre.2017.11.008

3. Xu C, Qu X. Cerium oxide nanoparticle: a remarkably versatile rare earth nanomaterial for biological applications. NPG Asia Mater. 2014;6:e90e90. doi:10.1038/am.2013.88

4. Khurana A, Anchi P, Allawadhi P, et al. Superoxide dismutase mimetic nanoceria restrains cerulein induced acute pancreatitis. Nanomedicine (Lond). 2019;14(14):1805-1825. doi:10.2217/nnm-2018-0318

5. Das S, Neal CJ, Ortiz J, Seal S. Engineered nanoceria cytoprotection in vivo: mitigation of reactive oxygen species and double-stranded DNA breakage due to radiation exposure. Nanoscale. 2018;10(45):21069-21075. doi:10.1039/C8NR04640A

6. Kim J, Kim HY, Song SY, et al. Synergistic oxygen generation and reactive oxygen species scavenging by manganese ferrite/ceria co-decorated nanoparticles for rheumatoid arthritis treatment. ACS nano. 2019;13(3):3206-3217. doi:10.1021/acsnano.8b08785

7. Yokel RA, Hussain S, Garantziotis S, Demokritou P, Castranova V, Cassee FR. The Yin: an adverse health perspective of nanoceria: uptake, distribution, accumulation, and mechanisms of its toxicity. Environ sci Nano. 2014;1(5):406-428. doi:10.1039/C4EN00039K

8. Konen-Adiguzel S, Ergene S. In vitro evaluation of the genotoxicity of $\mathrm{CeO} 2$ nanoparticles in human peripheral blood lymphocytes using cytokinesis-block micronucleus test, comet assay, and gamma H2AX. Toxicol Ind Health. 2018;34(5):293-300. doi:10.1177/0748233717753780

9. Eskandari N, Nejadi Babadaei MM, Nikpur S, et al. Biophysical, docking, and cellular studies on the effects of cerium oxide nanoparticles on blood components: in vitro. Int $J$ Nanomedicine. 2018;13:4575-4589. doi:10.2147/IJN.S172162

10. Hussain S, Al-Nsour F, Rice AB, et al. Cerium dioxide nanoparticles induce apoptosis and autophagy in human peripheral blood monocytes. ACS nano. 2012;6(7):5820-5829. doi:10.1021/nn302235u

11. Yokel RA, Tseng MT, Dan M, et al. Biodistribution and biopersistence of ceria engineered nanomaterials: size dependence. Nanomedicine. 2013;9 (3):398-407. doi:10.1016/j.nano.2012.08.002

12. Hirst SM, Karakoti A, Singh S, et al. Bio-distribution and in vivo antioxidant effects of cerium oxide nanoparticles in mice. Environ Toxicol. 2013;28(2):107-118. doi:10.1002/tox.20704

13. Nemmar A, Al-Salam S, Beegam S, Yuvaraju P, Ali BH. The acute pulmonary and thrombotic effects of cerium oxide nanoparticles after intratracheal instillation in mice. Int J Nanomedicine. 2017;12:2913-2922. doi:10.2147/IJN.S127180

14. Tseng MT, Lu X, Duan X, et al. Alteration of hepatic structure and oxidative stress induced by intravenous nanoceria. Toxicol Appl Pharmacol. 2012;260(2):173-182. doi:10.1016/j.taap.2012.02.008

15. Del Turco S, Ciofani G, Cappello V, et al. Effects of cerium oxide nanoparticles on hemostasis: coagulation, platelets, and vascular endothelial cells. J Biomed Mater Res A. 2019;107(7):1551-1562. doi:10.1002/jbm.a.36669

16. Sambrano GR, Weiss EJ, Zheng YW, Huang W, Coughlin SR. Role of thrombin Signalling in platelets in haemostasis and thrombosis. Nature. 2001;413(6851):74-78. doi:10.1038/35092573

17. Tucker KL, Sage T, Gibbins JM. Clot retraction. Methods Mol Biol. 2012;788:101-107.

18. Stabenfeldt SE, Gourley M, Krishnan L, Hoying JB, Barker TH. Engineering fibrin polymers through engagement of alternative polymerization mechanisms. Biomaterials. 2012;33(2):535-544. doi:10.1016/j.biomaterials.2011.09.079

19. Zucker RM, Daniel KM, Massaro EJ, Karafas SJ, Degn LL, Boyes WK. Detection of silver nanoparticles in cells by flow cytometry using light scatter and far-red fluorescence. Cytometry Part A. 2013;83(10):962-972. doi:10.1002/cyto.a.22342

20. Ibuki Y, Toyooka T. Nanoparticle uptake measured by flow cytometry. Methods Mol Biol. 2012;926:157-166.

21. Singh SK, Singh MK, Nayak MK, Kumari S, Grácio JJ, Dash D. Size distribution analysis and physical/fluorescence characterization of graphene oxide sheets by flow cytometry. Carbon. 2011;49(2):684-692. doi:10.1016/j.carbon.2010.10.020

22. Mazzolini J, Weber RJ, Chen HS, et al. Protein corona modulates uptake and toxicity of nanoceria via clathrin-mediated endocytosis. Biol Bull. 2016;231(1):40-60. doi:10.1086/689590

23. Manasa K, Vani R. Influence of oxidative stress on stored platelets. Adv Hematol. 2016;2016:4091461. doi:10.1155/2016/4091461

24. Masselli E, Pozzi G, Vaccarezza M, et al. ROS in platelet biology: functional aspects and methodological insights. Int J Mol Sci. 2020;21(14):4866. doi:10.3390/ijms21144866

25. Shrivastava S, Singh SK, Mukhopadhyay A, Sinha AS, Mandal RK, Dash D. Negative regulation of fibrin polymerization and clot formation by nanoparticles of silver. Colloids Surf B Biointerfaces. 2011;82(1):241-246. doi:10.1016/j.colsurfb.2010.08.048

26. Shrivastava S, Bera T, Singh SK, Singh G, Ramachandrarao P, Dash D. Characterization of antiplatelet properties of silver nanoparticles. ACS nano. 2009;3(6):1357-1364. doi:10.1021/nn900277t

27. Hajtuch J, Hante N, Tomczyk E, et al. Effects of functionalized silver nanoparticles on aggregation of human blood platelets. Int J Nanomedicine. 2019;14:7399-7417. doi:10.2147/IJN.S213499

28. Singh SK, Singh MK, Nayak MK, et al. Thrombus inducing property of atomically thin graphene oxide sheets. ACS nano. 2011;5(6):4987-4996. doi: $10.1021 / \mathrm{nn} 201092 \mathrm{p}$ 
29. Singh SK, Singh MK, Kulkarni PP, Sonkar VK, Gracio JJ, Dash D. Amine-modified graphene: thrombo-protective safer alternative to graphene oxide for biomedical applications. ACS nano. 2012;6(3):2731-2740. doi:10.1021/nn300172t

30. Woronowicz K, Dilks JR, Rozenvayn N, et al. The platelet actin cytoskeleton associates with SNAREs and participates in alpha-granule secretion. Biochemistry. 2010;49(21):4533-4542. doi:10.1021/bi100541t

31. Poulter NS, Thomas SG. Cytoskeletal regulation of platelet formation: coordination of F-actin and microtubules. Int J Biochem Cell Biol. 2015;66:69-74. doi:10.1016/j.biocel.2015.07.008

32. Shaydakov ME, Sigmon DF, Blebea J. Thromboelastography. Treasure Island (FL): StatPearls Publishing; 2021.

International Journal of Nanomedicine

\section{Publish your work in this journal}

The International Journal of Nanomedicine is an international, peer-reviewed journal focusing on the application of nanotechnology in diagnostics, therapeutics, and drug delivery systems throughout the biomedical field. This journal is indexed on PubMed Central, MedLine, CAS, SciSearch ${ }^{\circledR}$, Current Contents ${ }^{\mathbb{B}} /$ Clinical Medicine, Journal Citation Reports/Science Edition, EMBase, Scopus and the Elsevier Bibliographic databases. The manuscript management system is completely online and includes a very quick and fair peer-review system, which is all easy to use. Visit http:// www.dovepress.com/testimonials.php to read real quotes from published authors.

Submit your manuscript here: https://www.dovepress.com/international-journal-of-nanomedicine-journal 